\title{
Percent Change From Nadir in Sum of Products of Perpendicular Diameters
}

National Cancer Institute

\section{Source}

National Cancer Institute. Percent Change From Nadir in Sum of Products of

Perpendicular Diameters. NCI Thesaurus. Code C132265.

The most recently recorded sum of products of perpendicular diameters minus the lowest sum of diameters previously recorded divided by the lowest sum of products of perpendicular diameters previously recorded, multiplied by 100 . 